\title{
Optoelectronics and defect levels in hydroxyapatite by first-principles
}

\author{
Leon A. Avakyan, ${ }^{1,2, a)}$ Ekaterina V. Paramonova, ${ }^{3}$ José Coutinho, ${ }^{1}$ Sven Öberg, ${ }^{4}$ Vladimir S. Bystrov, ${ }^{3}$ and \\ Lusegen A. Bugaev ${ }^{2}$ \\ 1) Department of Physics and I3N, University of Aveiro, Campus Santiago, 3810-193 Aveiro, \\ Portugal \\ ${ }^{2)}$ Physics Faculty, Southern Federal University, Zorge street 5, Rostov-on-Don 344090 , \\ Russian Federation \\ ${ }^{3)}$ Institute of Mathematical Problems of Biology, Keldysh Institute of Applied Mathematics, \\ Russian Academy of Sciences, Vitkevicha street 1, Pushchino, 142290, Moscow region, \\ Russian Federation \\ ${ }^{4)}$ Department of Engineering Sciences and Mathematics, Luleå University of Technology, SE-97187 Luleå, \\ Sweden
}

Hydroxyapatite (HAp) is an important component of mammal bones and teeth, being widely used in prosthetic implants. Despite the importance of HAp in medicine, several promising applications involving this material (e.g. in photo-catalysis), depend on how well we understand its fundamental properties. Among the ones that are either unknown or not known accurately we have the electronic band structure and all that relates to it, including the band gap width. We employ state-of-the-art methodologies, including density hybrid-functional theory and many-body perturbation theory within the GW approximation, to look at the optoelectronic properties of HAp. These methods are also applied to the calculation of defect levels. We find that the use of a mix of (semi-)local and exact exchange in the exchange-correlation functional, brings a drastic improvement to the band structure. Important side-effects include improvements in the description of dielectric and optical properties, not only involving conduction band (excited) states, but also the valence. We find that the highly dispersive conduction band bottom of HAp originates from anti-bonding $\sigma^{*}$ states along the $\cdots \mathrm{OH}-\mathrm{OH}-\cdots$ infinite chain, suggesting the formation of a conductive 1D-ice phase. The choice of the exchange-correlation treatment to the calculation of defect levels was also investigated by using the OH-vacancy as testing-model. We find that donor and acceptor transitions obtained within semi-local DFT differ from those of hybrid-DFT by almost $2 \mathrm{eV}$. Such a large discrepancy emphasizes the importance of using a high-quality description of the electron-electron interactions in the calculation of electronic and optical transitions of defects in HAp. Published in Journal of Applied Physics 148, 154706 (2018). https://doi.org/10.1063/1.5025329

\section{INTRODUCTION}

Ceramics based on calcium apatites are important materials in health care, biology, ecology and catalysis. ${ }^{1,2}$ Among the apatites, hydroxyapatite (HAp) is singled out as the main component of vertebrate bones and teeth. Due to its innate bio-activity, HAp became a widely used biomaterial in medicine, with applications on bone and dental implants (for instance as filler and coating material) ${ }^{3-5}$ as well as an absorbent for liquid chromatography. ${ }^{6,7}$

Much of the emerging interest on HAp and related materials stems from their photo-catalytic and ferroelectric properties. ${ }^{8 ?, 9}$ Ultra-violet (UV) assisted filtration and decomposition of pharmaceutical pollutants, dyes and bacteria inactivation have been demonstrated. ${ }^{10-16}$ For these applications, modified HAp-based materials or composites, such as Ti-doped HAp, ${ }^{9} \mathrm{TiO}_{2}$-supported HAp, ${ }^{12,15}$ graphene-oxide supported HAp, ${ }^{16}$ as well as thermally modified material, ${ }^{10,13,14}$ are the most promising.

The origin of the photocatalytic activity of HAp has been attributed to the production of superoxide $\mathrm{O}_{2}^{\bullet-} \mathrm{rad}-$ icals, following the absorption of UV light by near-surface

a)Electronic mail: laavakyan@sfedu.ru vacancies and subsequent electron transfer to oxygen in the atmosphere. ${ }^{6,10,17}$ These observations were recently supported by some of us by means of density functional calculations. $^{18}$

In order to have an accurate picture of the photocatalytic mechanisms taking place, it is crucial that we are provided with accurate figures for the fundamental properties of the material, as well as methodologies to estimate them. These include, the crystalline structure, phonon dispersion, electronic band structure, dielectric response, electronic band gap, among others. Due to poor sample quality, most of these quantities are associated with large error bars. For instance, the measured width of the forbidden electronic gap $\left(E_{\mathrm{g}}\right)$ ranges from above $6 \mathrm{eV}$ down to $3.95 \mathrm{eV} .{ }^{19,20}$ Analogously, and despite much progress on the theoretical side, density functional theory (DFT) based on a local or semi-local description of the exchange-correlation interactions between electrons, give $E_{\mathrm{g}}$ values between $4.5 \mathrm{eV}$ and $5.4 \mathrm{eV}$, also depending on the particular choice of basis type for describing the valence states. ${ }^{7,21-24}$ As prudently noted by the authors of Ref. 24, the inherent insufficiencies of a (semi-)local exchange-correlation approximation severely underestimate the band gap of insulators, and therefore, it is likely that for HAp the true value of $E_{\mathrm{g}}$ lies well above $5.5 \mathrm{eV}$.

Defect-free HAp is transparent to visible light and the above arguments suggest that electronic excitations are 
achievable only under middle- or far-ultraviolet illumination, most probably with $h \nu>6 \mathrm{eV}$. The report of Nishikawa and co-workers ${ }^{6}$ on the photocatalytic activity using a light source of $h \nu=4.88 \mathrm{eV}(\lambda=254 \mathrm{~nm})$, implies that behind this effect we should have a transition involving at least one bound state located in the gap, most probably associated with defects. Now the question is: Are we dealing with vacancies? Or should that be caused by other point defects, impurities, grain boundaries or even surfaces states? In previous studies several point defects in HAp were inspected by using the local density approximation (LDA) to the exchangecorrelation potential. It was found that an oxygen vacancy in the phosphate $\left(\mathrm{PO}_{4}\right)$ unit introduces a fully occupied one-electron level in the lower half of the gap. ${ }^{1,18}$ The gap of the defective system was claimed to be compatible with the photocatalytic threshold energy. However, the lack of physical significance of the Kohn-Sham eigenvalues along with large self-interaction errors from the LDA, ${ }^{25}$ makes any assignment of an optical transition to a defect based on its Kohn-Sham band structure, highly speculative.

We have therefore to (i) employ state-of-the-art techniques to deal with the exchange-correlation problem, and to (ii) use physically sound methodologies to obtain defect-related transitions. The first issue has been successfully addressed in wide-gap materials, by admixing a fraction of exact exchange with the local or semilocal exchange. The exact exchange term is obtained from Hartree-Fock theory, it is fully non-local and free from electronic self-interactions. The resulting functionals have been termed hybrid and in general they improve molecular ionization energies and affinities, as well as the description of the band structure of solids, particularly of wide-gap insulators. ${ }^{26-31}$ The second issue must be addressed by calculating defect transitions solely based on the many-body energy obtained from the density functional. Two methods have been widely used, namely from the defect formation energy as function of its charge state, ${ }^{32}$ or by comparing defect ionization energies and electron affinities (still ground state properties) with analogous quantities calculated for defects with well characterized levels. ${ }^{33}$

We also note that since the underestimation of the gap affects the one-electron contribution to the total energy, it will also affect the calculation of quantities that strongly depend on the quality of the band structure, like migration barriers, work-functions, dielectric response, among many others. For instance, Corno et al. ${ }^{34}$ investigated the structural and vibrational properties of HAp using the B3LYP hybrid exchange functional. ${ }^{27}$ Not surprisingly, they reported $E_{\mathrm{g}}=7.9 \mathrm{eV}$, about $2.5 \mathrm{eV}$ wider than the LDA and the generalized gradient approximated (GGA) results.

The availability of alternative hybrid exchangecorrelation functionals, combined with the urgency of finding appropriate methodologies to study defects in HAp and related materials, are the main motivations be- hind this work. Below we present a comparative study of different properties, including the band structure, optical properties and defect formation energies as function of the Fermi energy, employing three popular hybrid functionals, namely: the PBE $0,{ }^{29} \mathrm{~B}^{2} \mathrm{LYP}^{28}$ and HSE06. ${ }^{31}$ The results are compared with quasi-particle band structure calculations obtained using the highly accurate Green's function method with screened interactions $(G W){ }^{35-37}$

\section{COMPUTATIONAL DETAILS}

\section{A. Semi-local and hybrid density functional calculations}

Calcium apatites have the general chemical formula $\mathrm{Ca}_{5}\left(\mathrm{PO}_{4}\right)_{3} X$, with $X$ being an electronegative element such as fluorine, chlorine or the hydroxyl $(\mathrm{OH})$ group. In the latter case the resulting material is hydroxyapatite. ${ }^{38}$ HAp solidifies in the form of an ionic molecular crystal, either of hexagonal $\left(P 6_{3} / m\right)$ or monoclinic $\left(P 2_{1} / b\right)$ symmetry (\#176 or \#14 in the International Tables for Crystallography, respectively ${ }^{39}$ ), whose unit cells enclose two or four formula units, respectively. ${ }^{40-42}$ For the $P 6_{3} / \mathrm{m}$ phase, hydroxyl units show a stochastic orientation. Thus, from x-ray data (macroscopic perspective), this effectively makes the material mirror-symmetric along the main axis. Conversely, when all $\mathrm{OH}$ units show the same alignment along the hexagonal axis, the mirror plane is lost and the space-group symmetry lowers to $P 6_{3}$.

The crystalline structure of HAp ( $P 6_{3}$ symmetry) is depicted in Fig. 1, where atoms belonging to one of the formula units are colored. A total of two and four inequivalent calcium and oxygen sites are found in HAp, respectively. The $\mathrm{Ca}_{\mathrm{I}}^{2+}$ cation columns are surrounded by $\mathrm{O}_{\mathrm{I}}$ and $\mathrm{O}_{\mathrm{II}}$ from $\mathrm{PO}_{4}^{3-}$ anion groups, while mirrorsymmetric $\mathrm{O}_{\mathrm{III}}$ sites and $\mathrm{Ca}_{\mathrm{II}}^{2+}$ ions form a hexagonal channel enclosing the hydroxyl anions. As mentioned above, different alignments of $\mathrm{OH}$ dipoles in the hydroxyl channels lead to different HAp phases. For instance, we may have i) a hexagonal disordered phase, with random orientations of $\mathrm{OH}$ dipoles; ii) a hexagonal ordered phase, where $\mathrm{OH}$ dipoles are all oriented along the same direction, or iii) a monoclinic phase, made of two adjacent cells along a basal lattice vector, with the first possessing a $\mathrm{OH}-\mathrm{OH}-\ldots$ column, while the second one showing an opposite HO-HO-... ordering. ${ }^{41}$ The last case shows anti-ferroelectric properties ${ }^{43}$ and the symmetry decreases to monoclinic $\left(P 2_{1} / b\right)$. Given that the electronic band structure of $P 6_{3^{-}}$and $P 2_{1} / b$-symmetric HAp are rather similar, ${ }^{24}$ the impact of flipping hydroxyl molecules on the properties under scrutiny is expected to be minor. Hence, we limited our analysis to the ordered hexagonal phase, allowing us to focus on the effect of the exchange-correlation treatment.

The calculations were carried out using density functional theory (VASP package) within the Kohn-Sham formalism. ${ }^{44-47}$ We employed the projector augmented- 


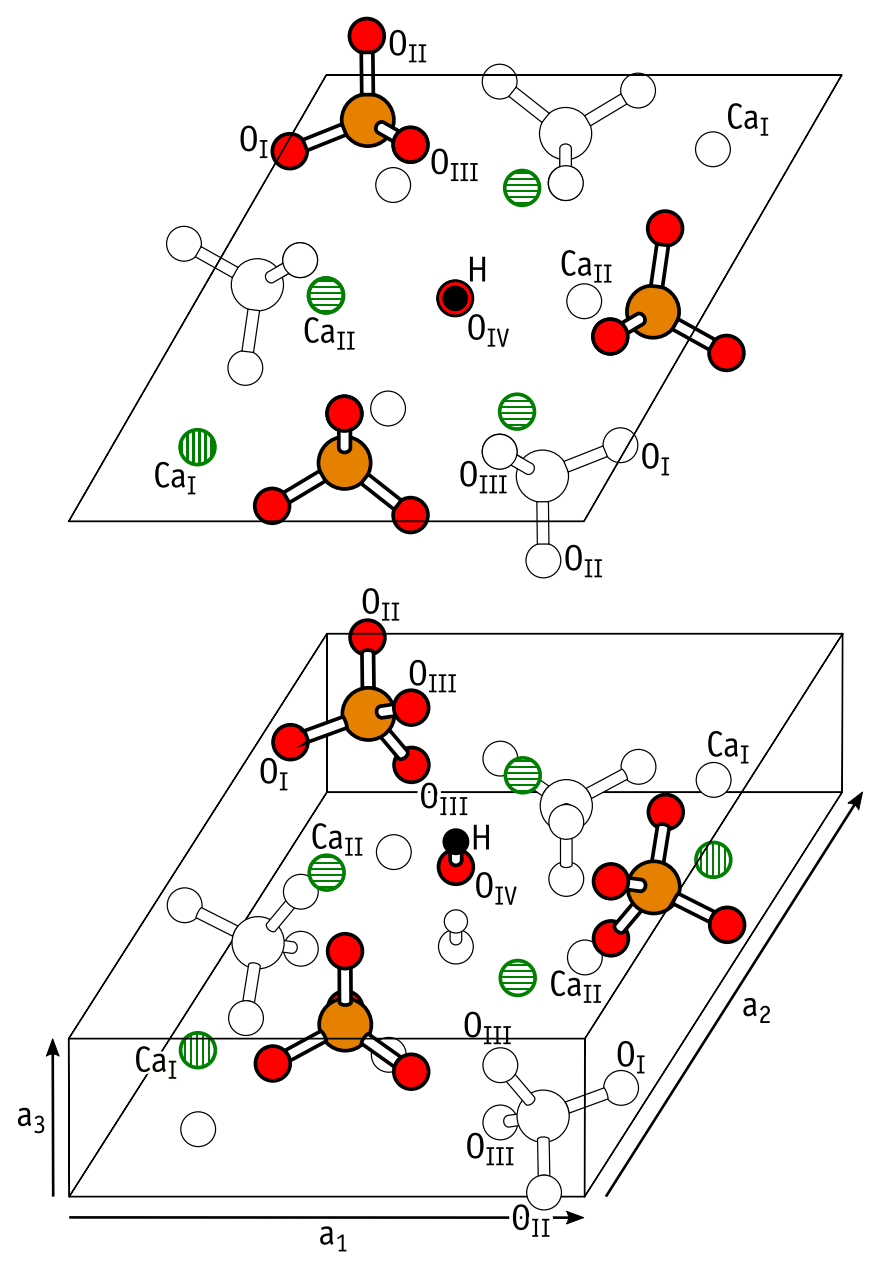

Figure 1. Top-projection and three-dimensional representation of a HAp unit cell centered on the hexagonal channel enclosing the $\mathrm{OH}$ molecular column. Only atoms from one $\mathrm{Ca}_{5}\left(\mathrm{PO}_{4}\right)_{3}(\mathrm{OH})$ molecular unit are colored. Phosphorus, oxygen calcium and hydrogen are shown in orange, red, hatched-green and black, respectively. Two inequivalent Ca atoms and four inequivalent $\mathrm{O}$ atoms are distinguished. Each phosphate molecule has two inequivalent $\mathrm{O}_{\mathrm{I}}$ and $\mathrm{O}_{\text {II }}$ sites and two equivalent $\mathrm{O}_{\text {III }}$ sites. Lattice parameters are also indicated.

wave (PAW) method ${ }^{48}$ to account for $[\mathrm{Ca}]: 1 \mathrm{~s}^{2} 2 \mathrm{~s}^{2} 2 \mathrm{p}^{6} 3 \mathrm{~s}^{2}$, $[\mathrm{P}]: 1 \mathrm{~s}^{2} 2 \mathrm{~s}^{2} 2 \mathrm{p}^{6}$ and $[\mathrm{O}]: 1 \mathrm{~s}^{2}$ core electrons. Valence electrons were described using plane waves with kinetic energy up to $E_{\text {cut }}=400 \mathrm{eV}$. Convergence tests to the basis quality were carried out by further increasing $E_{\text {cut }}$ and monitoring the change of the OH flipping energy. This is obtained by subtracting the energy $E_{\mathrm{OH}-\mathrm{OH}}$ of the unit cell shown in Fig. 1 (with an OH-OH hydroxyl alignment) to $E_{\mathrm{OH}-\mathrm{HO}}$ from a cell with anti-parallel $\mathrm{OH}-$ HO alignment. The results obtained within the GGA to the exchange-correlation are depicted in Fig. 2(a). For $E_{\text {cut }}=600 \mathrm{eV}$ the difference $E_{\mathrm{OH}-\mathrm{HO}}-E_{\mathrm{OH}-\mathrm{OH}}$ is $0.387 \mathrm{eV}$. We also conclude that all $E_{\text {cut }}$ values give essentially the same answer, which is close to $0.39 \mathrm{eV}$ per unit cell with an error bar of about $50 \mathrm{meV}(\sim 1 \mathrm{meV} /$ atom $)$

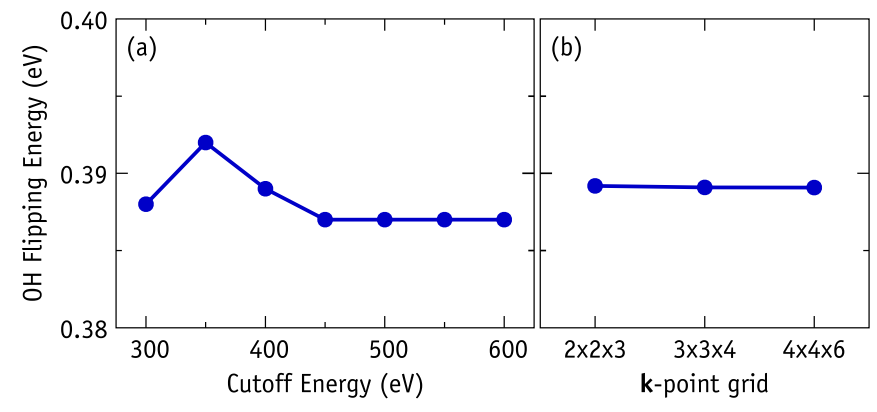

Figure 2. Convergence tests showing the energy difference between two HAp unit cells with $\mathrm{OH}-\mathrm{OH}$ and $\mathrm{OH}-\mathrm{HO}$ hydroxyl group alignments as a function of the plane wave cut-off energy (a), and as a function of the k-point sampling grid with $E_{\text {cut }}=400 \mathrm{eV}$ (b).

only. The results are close to $0.4 \mathrm{eV}$ as obtained in Refs. 24 and 23 using $E_{\text {cut }}$ values of $700 \mathrm{eV}$ and $500 \mathrm{eV}$, respectively. They are also compatible with the experimental estimate for the flipping activation energy of $0.86 \mathrm{eV}$ (which can be interpreted as approximately $E_{\mathrm{OH}-\mathrm{HO}}-E_{\mathrm{OH}-\mathrm{OH}}$ plus a reverse-flipping barrier). ${ }^{49} \mathrm{Fi}-$ nally, we confirmed that increasing $E_{\text {cut }}$ from $400 \mathrm{eV}$ to $600 \mathrm{eV}$ led to a negligible improvement to the band structure. For instance, the highest occupied and lowest occupied state energies changed by less than $8 \mathrm{meV}$.

The many-body electronic potential was evaluated using either the GGA according to Perdew, Burke, Ernzerhof (PBE), ${ }^{50}$ or one of the following hybrid density functionals: (i) The HSE06 of Heyd, Scuseria and Ernzerhof; ${ }^{30,31}$ (ii) The Becke three-parameter (B3LYP) functional; ${ }^{27}$ (iii) The PBE0 functional. ${ }^{29}$ While the exact-exchange contribution in B3LYP was originally adjusted in order to reproduce experimental atomic energetics data, for the case of HSE06 and PBE0 functionals, a fraction $\alpha=1 / 4$ of Fock exchange is considered, and that is based on the adiabatic connection formula.

The Brillouin zone (BZ) was sampled using a $\Gamma$ centered $2 \times 2 \times 3$ mesh of k-points. Figure $2(\mathrm{~b})$ shows no noticeable improvements in the quality of the results upon increasing the sampling density. The Hartree-Fock exact exchange was evaluated at the same $\mathbf{k}$-point grid used for the DFT potential, and stored on a real-space grid of $128 \times 128 \times 96$ points along $\mathbf{a}_{1}, \mathbf{a}_{2}$ and $\mathbf{a}_{3}$ lattice vectors, respectively, c.f. Fig. 1. The experimental lattice constants are $a_{1}=a_{2}=a=9.417 \AA$ and $a_{3}=c=6.875 \AA,{ }^{42}$ corresponding to a grid density of about 14 points/ $\AA$ along all three directions.

The calculations of HAp properties were performed on fully relaxed cells. Lattice relaxations were performed using a quasi-Newton algorithm combined with an improved basis and real space potential/density grid $\left(E_{\text {cut }}=520 \mathrm{eV}\right.$ and a grid comprising $168 \times 168 \times 128$ points). The relaxation cycle was stopped when the maximum force acting on lattice vectors and ions became less than $5 \mathrm{meV} / \AA$. 


\section{B. Dielectric response}

The static macroscopic dielectric tensor $\boldsymbol{\epsilon}_{\mathrm{s}}$ couples a time-independent uniform electric field $\mathcal{E}$ to the polarization $\mathbf{P}$ of a material. The polarization may have different microscopic origins, such as electronic, ionic, orientational and from space-charge mechanisms. The last two processes are more important for liquid systems, where polar molecules can rotate and diffuse rather easier than in solids. Moreover, these mechanisms are temperaturedependent and are hardly considered within the DFT framework. Here we consider the electronic polarization within the adiabatic approximation, as well as polarization effects due to ionic vibrations (phonons). Although the flipping of $\mathrm{OH}$ units are likely to affect the dielectric response of HAp, such calculations are outside the scope of the present study. In the following, the dielectric tensor corresponding to the contribution of the electronic subsystem (ion-clamped) is denoted as $\boldsymbol{\epsilon}_{\infty}$, while the ionic contribution is denoted as $\epsilon_{\mathrm{ph}}$.

The calculation of $\boldsymbol{\epsilon}_{\infty}$ was carried out using the polarization expansion after discretization (PEAD) approach of Nunes and Gonze. ${ }^{51}$ The procedure follows from the application of a finite homogeneous electric field $\mathcal{E}$ by introducing a set of field-polarized Bloch functions $\left\{\psi^{\mathcal{E}}\right\}$ in the Kohn-Sham machinery, and minimizing the electric enthalpy functional,

$$
E\left[\left\{\psi^{\mathcal{E}}\right\}, \mathcal{E}\right]=E_{0}\left[\left\{\psi^{\mathcal{E}}\right\}\right]-\Omega \mathcal{E} \cdot \mathbf{P}\left[\left\{\psi^{\mathcal{E}}\right\}\right],
$$

where $E_{0}$ is the standard (zero-field) density functional, $\mathbf{P}$ is the macroscopic polarization according to the "modern theory of polarization" 52 and $\Omega$ is the cell volume. Accordingly, the ion-clamped macroscopic dielectric tensor elements are obtained as

$$
\epsilon_{\infty, i j}=\delta_{i j}+4 \pi \frac{\partial P_{i}}{\partial \mathcal{E}_{j}}
$$

with $i$ and $j$ representing Cartesian directions. The method accounts for local-field effects (DFT or hybridDFT level) in a natural manner through the selfconsistent procedure. Further details on the PEAD method and its implementation can be found elsewhere. ${ }^{53}$

The Born effective charge tensors $\mathbf{Z}_{\alpha}^{*}$ are also by obtained as a side-product. These couple the applied electric field to the induced force $\mathbf{F}_{\alpha}$ acting on the $\alpha$-th atom. Hence,

$$
Z_{\alpha, i j}^{*}=\frac{1}{e} \frac{\partial F_{\alpha, j}}{\partial \mathcal{E}_{i}}=\frac{\Omega}{e} \frac{\partial P_{i}}{\partial u_{\alpha, j}},
$$

is obtained from finite-differences, where $u_{\alpha, j}$ is a sublattice atomic displacement of the $\alpha$-th atom along $j$ and $e$ is the elementary charge. In our calculations we employed atomic displacements of $0.015 \AA$ and the magnitude of the electric field was set to $1 \mathrm{meV} / \AA$. Test calculations within PBE-level showed numerical stability with electric field magnitudes of up to $10 \mathrm{meV} / \AA$.
The phonon contribution to the macroscopic dielectric tensor, $\boldsymbol{\epsilon}_{\mathrm{ph}}$, can be obtained from the Born effective charges and dynamical matrix elements (second order derivatives of the energy with respect to ionic positions). The latter was calculated using finite differences with the atoms being displaced by $0.015 \AA$ along symmetry-irreducible directions. The eigenvalues $\omega_{\lambda}^{2}$ (with $\lambda=1, \ldots, 3 N$ ) of a dynamical matrix involving $N$ ions correspond to phonon frequencies $\omega_{\lambda}$ at the BZ center $(\mathbf{q}=0)$. Their respective eigenvectors are the normal modes of vibration $A_{\lambda, \alpha, i}$, which embody the phonon polarization. According to classical dispersion theory, ${ }^{54,55}$ the $\boldsymbol{\epsilon}_{\mathrm{ph}}$ tensor elements are given by

$$
\epsilon_{\mathrm{ph}, i j}=\sum_{\omega_{\lambda}^{2}>0} \frac{\tilde{Z}_{\lambda, i}^{\star} \tilde{Z}_{\lambda, j}^{\star}}{\Omega \omega_{\lambda}^{2}}
$$

with $\tilde{Z}_{\lambda, i}^{\star}=\sum_{\alpha, j} Z_{\alpha, i j}^{\star}\left(M_{\alpha}\right)^{-\frac{1}{2}} A_{\lambda, \alpha, j}$ being vibrational mode effective charges, where $M_{\alpha}$ is the mass of the $\alpha$-th ion. The phonon contribution to the dielectric tensor was calculated within PBE-level only.

The static dielectric properties of HAp were measured on crystallite powder samples, ${ }^{43,56,57}$ so they effectively behave as macroscopically isotropic. In order to compare the calculations with the observations we make use of the effective medium approximation (EMA), ${ }^{58,59}$ which defines an orientational-averaged dielectric constant $\epsilon_{\mathrm{S} \text {, EMA }}$ as

$$
\epsilon_{\mathrm{s}, \mathrm{EMA}}=\frac{1}{4}\left(\epsilon_{\mathrm{s}, \perp}+\sqrt{\epsilon_{\mathrm{s}, \perp}\left(\epsilon_{\mathrm{s}, \perp}+8 \epsilon_{\mathrm{s}, \|}\right)}\right)
$$

with axial $\epsilon_{\mathrm{s}, \|}=\epsilon_{\mathrm{s}, z z}$ and basal $\epsilon_{\mathrm{s}, \perp}=\epsilon_{\mathrm{s}, x x}=\epsilon_{\mathrm{s}, y y}$ elements being parallel and perpendicular to the $c$-axis of the hexagonal lattice, respectively.

The electronic component of the frequency-dependent macroscopic dielectric tensor, $\boldsymbol{\epsilon}_{\infty}(\omega)$, characterizes the response to a long-wave length field (in the limit $\mathbf{q} \rightarrow$ $0)$ with frequency $\omega$. We obtained $\epsilon_{\infty}(\omega)$ within the independent particle picture as derived by Adler and Wiser. ${ }^{60,61}$ Accordingly, the imaginary part of the dielectric tensor, $\boldsymbol{\epsilon}_{\infty}^{(2)}(\omega)$, is ${ }^{62}$

$$
\begin{array}{r}
\epsilon_{i j}^{(2)}(\omega)=\frac{4 \pi^{2} e^{2}}{\Omega} \lim _{\mathbf{q} \rightarrow 0} \frac{1}{q^{2}} \sum_{n, n^{\prime}, \mathbf{k}} 2 w_{\mathbf{k}} \delta\left(E_{n \mathbf{k}}-E_{n^{\prime} \mathbf{k}}-\hbar \omega\right) \times \\
\left\langle u_{n, \mathbf{k}+\mathbf{e}_{i} q} \mid u_{n^{\prime}, \mathbf{k}}\right\rangle\left\langle u_{n^{\prime}, \mathbf{k}} \mid u_{n, \mathbf{k}+\mathbf{e}_{j} q}\right\rangle, \quad(6)
\end{array}
$$

where $E_{n \mathbf{k}}$ and $E_{n^{\prime} \mathbf{k}}$ are the Kohn-Sham eigenvalues of filled and empty states $\psi_{n \mathbf{k}}$ and $\psi_{n^{\prime} \mathbf{k}}$, respectively, with wave vector $\mathbf{k}$ within the BZ. The quantities $\left|u_{n, \mathbf{k}+\mathbf{e}_{i} q}\right\rangle$ are obtained using density-functional perturbation theory, ${ }^{62}$ and represent the first-order change of the cell-periodic part of the wave function $\psi_{n \mathbf{k}+\mathbf{q}}$ with respect to $\mathbf{q}$, where $\mathbf{e}_{i}$ are unit vectors for the three Cartesian directions. Finally, the real part of the dielectric 
tensor, $\boldsymbol{\epsilon}^{(1)}(\omega)$, is obtained by the usual Kramers-Kronig transformation.

The number of empty states and k-points in the summation of Eq. 6 should be large enough to produce converged results. Tests were performed within PBE-level, from which we concluded that a $4 \times 4 \times 6 \mathrm{k}$-point mesh and a total of 400 bands (266 empty states) were enough to reach convergence of $\boldsymbol{\epsilon}_{\infty}(\omega)$ up to $\omega=30 \mathrm{eV}$.

\section{Many-body perturbation theory calculations}

We also performed many-body perturbation calculations where the self-energy was accounted for within the $G W$ approximation (see Refs. 37, 63, and 64). This allowed us to benchmark the description of the HAp band structure, and by comparison, to assess the performance of DFT and hybrid-DFT methods. Unlike standard den- sity functional theory, the $G W$ method accounts for the many-body electron-electron interactions via screening of the exchange interactions with the frequency-dependent microscopic dielectric matrix. In practice, quasi-particle energies can be calculated within the spirit of first-order perturbation theory by solving the quasi-particle equation

$$
E_{n \mathbf{k}}^{\mathrm{QP}}=\operatorname{Re}\left[\left\langle\psi_{n \mathbf{k}}\left|T+V_{\mathrm{ne}}+V_{\mathrm{H}}+\Sigma\left(E_{n \mathbf{k}}\right)\right| \psi_{n \mathbf{k}}\right\rangle\right],
$$

where $T$ is the kinetic energy operator, $V_{\text {ne }}$ accounts for the nuclear-electron interactions and $V_{\mathrm{H}}$ is the Hartree potential. DFT-PBE eigenvalues and wave functions $E_{n \mathbf{k}}$ and $\psi_{n \mathbf{k}}$, respectively, were used in Eq. 7 in order to produce single-shot $\left(G_{0} W_{0}\right)$ quasi-particle energies. Diagonal elements of the self-energy matrix are given by

$$
\begin{aligned}
\left\langle\psi_{n \mathbf{k}}|\Sigma(\omega)| \psi_{n \mathbf{k}}\right\rangle= & \frac{1}{\Omega} \sum_{\mathbf{q} \mathbf{G G}^{\prime}} \sum_{n^{\prime}} \frac{i}{2 \pi} \int_{-\infty}^{+\infty} d \omega^{\prime} W\left(\mathbf{G}+\mathbf{q}, \mathbf{G}^{\prime}+\mathbf{q}, \omega^{\prime}\right) \\
& \times \frac{\left\langle\psi_{n \mathbf{k}}\left|e^{i(\mathbf{G}+\mathbf{q}) \cdot \mathbf{r}}\right| \psi_{n^{\prime} \mathbf{k}-\mathbf{q}}\right\rangle\left\langle\psi_{n^{\prime} \mathbf{k}-\mathbf{q}}\left|e^{-i\left(\mathbf{G}^{\prime}+\mathbf{q}\right) \cdot \mathbf{r}^{\prime}}\right| \psi_{n \mathbf{k}}\right\rangle}{\omega+\omega^{\prime}-E_{n^{\prime} \mathbf{k}-\mathbf{q}}+i \eta \operatorname{sign}\left(E_{n^{\prime} \mathbf{k}-\mathbf{q}}-E_{\mathrm{F}}\right)}
\end{aligned}
$$

where $E_{\mathrm{F}}$ is the Fermi energy and $\eta$ an ubiquitous but vanishing complex shift. The dynamically screened Coulomb potential, $W$, was calculated within the random-phase approximation (RPA), which essentially employs the Hartree potential to account for the local field effects. The summations in Eq. 8 ran over a grid of vectors $\mathbf{G}+\mathbf{q}$, whose magnitude was limited by a cut-off energy $E_{\text {cut }}^{G W}=100 \mathrm{eV}$, whereas the band index $n^{\prime}$ went up to 1344 bands (1210 of them were empty). The response function was evaluated considering electronic transitions between states on a $\Gamma$ centered $4 \times 4 \times 6$ k-point grid (as described above for the macroscopic dielectric tensor), while the momentum transfer vectors q were restricted to a coarser $2 \times 2 \times 3$ grid. Convergence tests using a denser $4 \times 4 \times 6$ q-grid, $E_{\text {cut }}^{G W}=150 \mathrm{eV}$ and 1410 bands showed no appreciable changes in the screened Coulomb potential. Finally, the dielectric function was also calculated from the polarizability considering the quasi-particle band structure energies.

\section{Formation energy of defects}

The procedure for the calculation of formation energies was described by Qian, Martin and Chadi when investigating the stability of GaAs surfaces. ${ }^{32}$ Accordingly, the energy needed to introduce a defect in a crystal (defect formation energy) is $E_{\mathrm{f}}=E-\sum_{i} n_{i} \mu_{i}-\Delta n_{\mathrm{e}} \mu_{\mathrm{e}}$, where $E$ is the total energy of the defective crystal made of $n_{i}$ atomic elements of species $i$ with chemical potential $\mu_{i}$. This formalism also considers the formation of defects with an excess of electrons $\Delta n_{\mathrm{e}}$ (with respect to the neutral state) when they are able to trap/release electrons from/to an electron reservoir with chemical potential $\mu_{\mathrm{e}}$.

Chemical potentials $\mu_{i}$ can vary within a limited range,

$$
\sum n_{i}^{\phi}\left(\mu_{i}-\mu_{i}^{0}\right) \leq \Delta H_{\mathrm{f}}^{\phi},
$$

with the upper limit taking place when the abundance of species $i$, pressure and temperature conditions are such that HAp becomes unstable against segregation of a compound $\phi$ made of $n_{i}^{\phi}$ elements of species $i$ and with heat of formation $\Delta H_{\mathrm{f}}^{\phi}$. In Eq. 9 the quantities $\mu_{i}^{0}$ are chemical potentials of standard phases (for HAp they stand for the energy per atom in black phosphorous, molecular oxygen, calcium metal and molecular hydrogen).

In the calculation of $\mu_{i}^{0}$ related to $\mathrm{O}_{2}$ and $\mathrm{H}_{2}$ molecules, we could add a term $k_{\mathrm{B}} T \cdot \ln p_{i}^{0}$ in order to account for the partial pressure $p_{i}^{0}$ of the gas source at temperature $T{ }^{23}$ Here, $k_{\mathrm{B}}$ is the Boltzmann constant. We also note that we are neglecting the contribution of entropy, although this assumption may be questionable, especially at high temperatures. Since we are interested in assessing the effect of the exchange-correlation treatment to the formation energy of defects, it is actually preferable to leave the results free of temperature and pressure effects. Note that the location of the electronic levels within the band gap does not depend on the actual choice of $\mu_{i}$ values.

Also from Eq. 9 we may relate the chemical potentials 
$\mu_{i}$ in the HAp solid with the heat of formation per HAp formula unit as,

$$
\sum n_{i}^{\mathrm{HAp}}\left(\mu_{i}-\mu_{i}^{0}\right)=\Delta H_{\mathrm{f}}^{\mathrm{HAp}}
$$

where the equality sign follows from the equilibrium established between the HAp solid phase and its own elements. The calculated heat of formation of HAp per formula unit within PBE and B3LYP levels is $-125.53 \mathrm{eV}$ and $-136.19 \mathrm{eV}$, respectively. The latter is in close agreement with $\Delta H_{\mathrm{f}}^{\mathrm{HAp}}=-138.88 \mathrm{eV}$ as determined by reaction-solution calorimetry measurements. ${ }^{65}$

When using periodic boundary conditions, a more convenient expression for the defect formation energy is obtained by referring to the number of atoms $\Delta n_{i}$ of species $i$ added to $\left(\Delta n_{i}>0\right)$ or removed from $\left(\Delta n_{i}<0\right)$ a pristine supercell made of $N^{\mathrm{HAp}}$ formula units with energy $\mu^{\mathrm{HAp}}=\sum_{i} n_{i}^{\mathrm{HAp}} \mu_{i}$

$$
E_{\mathrm{f}}=E-N^{\mathrm{HAp}} \mu^{\mathrm{HAp}}-\sum_{i} \Delta n_{i} \mu_{i}+q\left(E_{\mathrm{v}}+E_{\mathrm{F}}\right),
$$

where $q$ is an integer referring to the charge state of the defect $\left(q=-\Delta n_{\mathrm{e}}\right.$ is positive/negative when defect levels within the gap are depleted/filled with $q$ electrons with respect to the neutral state). The electronic chemical potential is also conveniently expressed by invoking the Fermi energy so that $\mu_{\mathrm{e}}=E_{\mathrm{v}}+E_{\mathrm{F}}$. The Fermi energy may vary between the valence band top (where $E_{\mathrm{F}}=0$ ) and conduction band bottom $\left(E_{\mathrm{F}}=E_{\mathrm{g}}\right)$. While the first and second terms in Eq. 11 can be readily obtained from a first-principles calculation, the last two terms can vary within certain limits imposed by the thermodynamic conditions. We also note that the third term only depends on defect-related species. Here we will look at the formation energy of a $\mathrm{OH}$ vacancy, so that we have to define chemical potentials for $\mathrm{O}$ and $\mathrm{H}$ only. We assume that the crystal is in equilibrium with $\mathrm{O}_{2}$ and $\mathrm{H}_{2}$ molecules (O- and H-rich conditions), so that $\mu_{\mathrm{O}}=\mu_{\mathrm{O}}^{0}$ and $\mu_{\mathrm{H}}=\mu_{\mathrm{H}}^{0}$. These were calculated from $\mathrm{O}_{2}$ (spin-1 state) and $\mathrm{H}_{2}$ molecules in a box of edge length $L=20 \AA$.

Finally, we note that charge neutrality has to be imposed to the whole cell of any periodic calculation. Hence, the introduction of a localized charge $q$ is accompanied by a uniform background counter-charge density $\rho_{\text {back }}=-q / \Omega .{ }^{68}$ The Coulomb interactions between the periodic array of charged defects and background are long-ranged, and are particularly strong for materials with low dielectric screening. To mitigate this effect we have to approximate the aperiodic total energy $E$ in Eq. 11 by a periodic total energy plus a correction, $\tilde{E}+E_{\text {corr }}$. While $\tilde{E}$ is the energy of the defective periodic supercell as obtained from first-principles, the charge correction was obtained according to the method proposed by Freysoldt, Neugebauer and Van de Walle $(\mathrm{FNV}),{ }^{69}$ which was recently generalized for anisotropic materials. ${ }^{70}$
Table I. Experimental (Exp.) and calculated ground state properties of HAp using different exchange-correlation functionals (PBE, HSE06, B3LYP and PBE0). Quantities shown are basal and axial lattice constants ( $a$ and $c$, respectively), bulk modulus $(B)$, pressure derivative of the bulk modulus $\left(B^{\prime}\right)$, ion-clamped $\left(\epsilon_{\infty}\right)$ and total $\left(\epsilon_{\mathrm{s}}\right)$ static dielectric constants along the direction perpendicular $(\perp$-subscripted $)$ and parallel (\|-subscripted) to the $c$-axis. Errors related to $B$ and $B^{\prime}$ were obtained from the fitting procedure. The table also shows the the effective medium approximated dielectric constant $\epsilon_{\mathrm{S}, \mathrm{EMA}}$, to be compared with the orientational-averaged data obtained from powder samples.

\begin{tabular}{lccccc}
\hline \hline Property & Exp. & PBE & HSE06 & B3LYP & PBE0 \\
\hline$a(\AA)$ & $9.417^{\mathrm{a}}$ & 9.537 & 9.481 & 9.577 & 9.477 \\
$c(\AA)$ & $6.875^{\mathrm{a}}$ & 6.909 & 6.923 & 6.877 & 6.851 \\
$B(\mathrm{GPa})$ & $89 \pm 1^{\mathrm{b}, \mathrm{c}}$ & $82 \pm 3$ & $83 \pm 1$ & $86 \pm 2$ & $82.8 \pm 0.3$ \\
$B^{\prime}$ & $6.9 \pm 0.6^{\mathrm{c}}$ & $4 \pm 2$ & $6 \pm 1$ & $4 \pm 2$ & $5.4 \pm 0.3$ \\
$\epsilon_{\infty, \perp}$ & - & 2.94 & 2.71 & 2.71 & 2.70 \\
$\epsilon_{\infty, \|}$ & - & 2.93 & 2.71 & 2.71 & 2.72 \\
$\epsilon_{\mathrm{s}, \perp}$ & - & 13.1 & 12.8 & 12.8 & 12.8 \\
$\epsilon_{\mathrm{S}, \|}$ & - & 8.8 & 8.2 & 8.2 & 8.2 \\
$\epsilon_{\mathrm{s}, \mathrm{EMA}}$ & $7-15^{\mathrm{d}}$ & 11.4 & 11.1 & 11.1 & 11.1 \\
\hline \hline
\end{tabular}

a Reference $^{42}$

b Reference ${ }^{66}$

${ }^{c}$ Reference ${ }^{67}$

d References ${ }^{43,56,57}$

\section{RESULTS AND DISCUSSION}

\section{A. Ground state properties of HAp}

We start by reporting on the structural and mechanical properties of HAp. Table I compares the calculated unit cell lattice parameters, bulk modulus, its pressure derivative and static dielectric properties, with respective experimental data. The table includes results obtained with PBE, HSE06, B3LYP and PBE0 exchangecorrelation functionals. The calculated lattice parameters within PBE-level are in line with previous PBE results (see Refs. 24 and 71 and references therein). They show the usual $\sim 1 \%$ overestimation with respect to the experimental data. This is known to be mostly due to an artificial over-delocalization of the electronic density when the GGA is employed. The B3LYP results improve on $c$ but $a$ is still overestimated by $\sim 1 \%$. This confirms previous reports that find B3LYP to generally overestimate the experimental lattice parameters as well. ${ }^{72} \mathrm{On}$ the other hand, our result differs from previous B3LYP calculations of HAp, where $a$ was underestimated by $1 \% .{ }^{34}$ This discrepancy is probably due to the use of a Gaussian-Bloch basis in Ref. 34. Atom-centered basis functions like these are expected to be less qualified to describe open-structure solids like HAp, where states overlapping void regions may be misrepresented. This will become more evident below, when discussing the 
electronic structure and screening properties.

Table I also indicates that the lattice parameters calculated within hybrid-DFT are generally closer to the experiments than those obtained using the semi-local functional. The best results are obtained for PBE0 with a deviation of less than $0.6 \%$ with respect to the experiments.

The Bulk modulus $(B)$ and its pressure derivative $\left(B^{\prime}\right)$ were obtained by fitting the Birch-Murnaghan equation of state, ${ }^{73}$

$$
E(\xi)=E_{0}+\frac{9 \Omega_{0} B}{2}\left[\xi^{2}+\left(B^{\prime}-4\right) \xi^{3}\right],
$$

to total energy data points $(E)$ at 8 different cell volumes $(\Omega)$ around equilibrium $\left(\Omega_{0}\right)$. The volume dependence enters in Eq, 12 as $\xi=\left[\left(\Omega_{0} / \Omega\right)^{2 / 3}-1\right] / 2$, and $E_{0}$ is the minimum energy at $\Omega_{0}$. The calculated bulk modulus and its pressure derivative show reasonable agreement with the experiments (see Table I). The errors reported on the table were obtained from the fitting procedure. B3LYP calculations deviates from the measurements by about $3 \%$ only, while other functionals underestimate the experimental value of $B$ by $7-8 \%$. This level of accuracy is in line with typical discrepancies found for many insulators. ${ }^{72}$

The ion-clamped static dielectric tensors $\boldsymbol{\epsilon}_{\infty}$ as obtained by the different hybrid-DFT schemes (see Table I) show little variation. They are about 0.2 lower than the PBE results. This is a property that depends on the ground-state density, and we interpret this difference as a consequence of the more diffuse PBE electron density. Interestingly, we find the electronic response of HAp to be essentially isotropic (within the error bar of the calculation methodology) with $\epsilon_{\infty, \perp} \approx \epsilon_{\infty, \|} \approx 2$.7. Such low anisotropy was also found for strontium apatites within the LDA. ${ }^{74}$ Also, the calculated low electronic screening is consistent with the open-structure and low atomic number of the HAp constituents.

The ionic contribution $\epsilon_{\mathrm{ph}}$ to $\boldsymbol{\epsilon}_{\mathrm{s}}$ was obtained solely within PBE and we found it considerably larger and more anisotropic than $\boldsymbol{\epsilon}_{\infty}$. Hence, the small functionaldependent fluctuations that affect the total dielectric constants $\left(\boldsymbol{\epsilon}_{\mathrm{s}}=\boldsymbol{\epsilon}_{\infty}+\boldsymbol{\epsilon}_{\mathrm{ph}}\right.$ in Table I) derive from variations in $\boldsymbol{\epsilon}_{\infty}$ only. Since the Born effective charges and phonon frequencies depend solely on the ground state density, not much improvement is anticipated if a hybrid-DFT method was used. From Eq. 4, we simply note that the calculation of $\epsilon_{\mathrm{ph}}$ at PBE-level is likely to be affected by the expected underestimation of the phonon frequencies (which is a characteristic of the PBE functional).

Although the measurement of individual $\epsilon_{\mathrm{s}, \perp}$ and $\epsilon_{\mathrm{s}, \|}$ components for HAp has not been reported, our values are not far from $\epsilon_{\mathrm{s}, \perp}=10.5$ and $\epsilon_{\mathrm{s}, \|}=8.7$ which were observed for crystalline fluorapatite. ${ }^{75}$ The effective medium approximated dielectric constant is estimated as $\epsilon_{\mathrm{S}, \mathrm{EMA}}=11$, regardless the exchange-correlation functional. The experimental static dielectric constant de- termined for HAp powder samples varies widely, and strongly depends on the porosity and amount of water within the samples. For example, according to the study of Ikoma et al., ${ }^{43} \epsilon_{\mathrm{s}}$ varies from 15 to 300 upon heating from 300 to $600 \mathrm{~K}$. On the other hand, Ref. 56 reports on the dielectric properties of Ca-doped HAp and arrives at $\epsilon_{\mathrm{s}}=6.75$ only, probably due to the pores in the ceramic pellets. The study of Hoepfner and Case $^{57}$ addresses precisely the issue of the porosity dependence of the dielectric constant for sintered HAp. By extrapolating the data to a vanishing porosity they arrived at $\epsilon_{\mathrm{s}} \approx 14$. This is closer, but somewhat above the calculated figure, possibly due to contamination of the samples.

\section{B. Electronic band structure}

Figures 3(a)-(d) compare the electronic band structure obtained using different exchange-correlation functionals (PBE, HSE06, B3LYP and PBE0) with the analogous $G_{0} W_{0}$ quasi-particle calculation shown in Figure 3(e). The band energies along the several high-symmetry directions were obtained by interpolation of the first-principles data using Wannier90. ${ }^{76}$

The shape of the PBE band structure in Figure 3(a) is indistinguishable from that reported by Slepko et al. ${ }^{24}$, displaying a low-dispersive valence band top and a highdispersive conduction band bottom (thick bands). Dispersion of the conduction band minimum states is considerably more pronounced along directions parallel to the $c$-axis $(\Gamma-A, K-H$, and $M-L)$, indicating a stronger carrier delocalization and mobility along the main axis. This property could be explored for tuning HAp electrical conductivity through n-type doping or for photo-current measurements. On the other hand, p-type doping is not expected to be beneficial. The valence band top states show very little dispersion, and their heavy holes imply a relatively lower mobility.

Also in agreement with Ref. 24 we find HAp to be an indirect-gap material with $E_{\mathrm{g}}=5.23 \mathrm{eV}$ at PBE-level. The conduction band minimum is located at $\mathbf{k}=\Gamma$, while the valence band top energy was found somewhere along $\Gamma-K$ or $\Gamma-M$. The valence band maximum along $\Gamma-M$ is only $0.1 \mathrm{meV}$ higher than the one along $\Gamma-K$. We note that this picture was the same regardless the functional used, including when using the $G_{0} W_{0}$ method.

The band structure obtained within HSE06-level is shown in Figure 3(b). The increase in the band gap width by more than $30 \%$ with respect to the PBE result is selfevident. Using HSE06 we obtain $E_{\mathrm{g}}=7.11 \mathrm{eV}$. Often, the band energies are offset in order to lock the valence band top at the origin of the energy scale. We did not follow this procedure, and that allowed us to disclose how the gap change depends on the shift of both valence band and conduction band states. Figures 3(b)-(d) show that admixing a fraction of Fock exchange with the semi-local exchange energy has a profound effect on both valence and conductions band states. Consequently, the use of 
(a) PBE

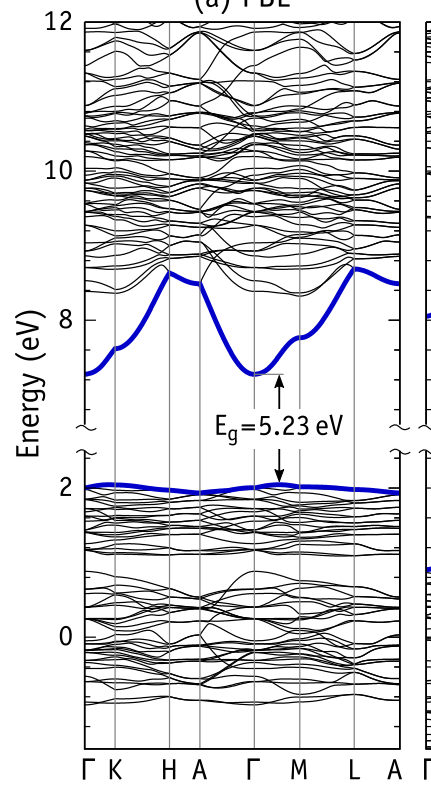

(b) HSEO6

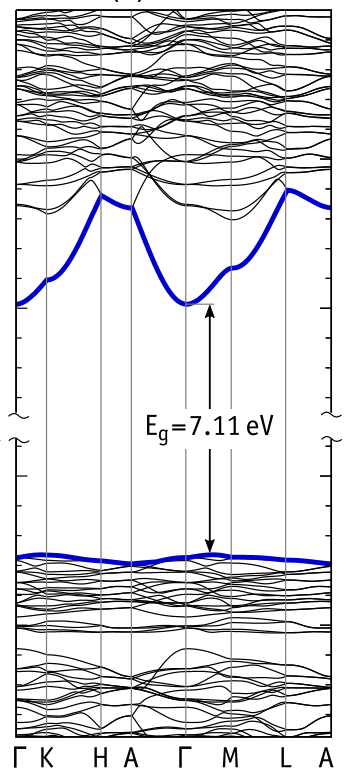

(c) B3LYP

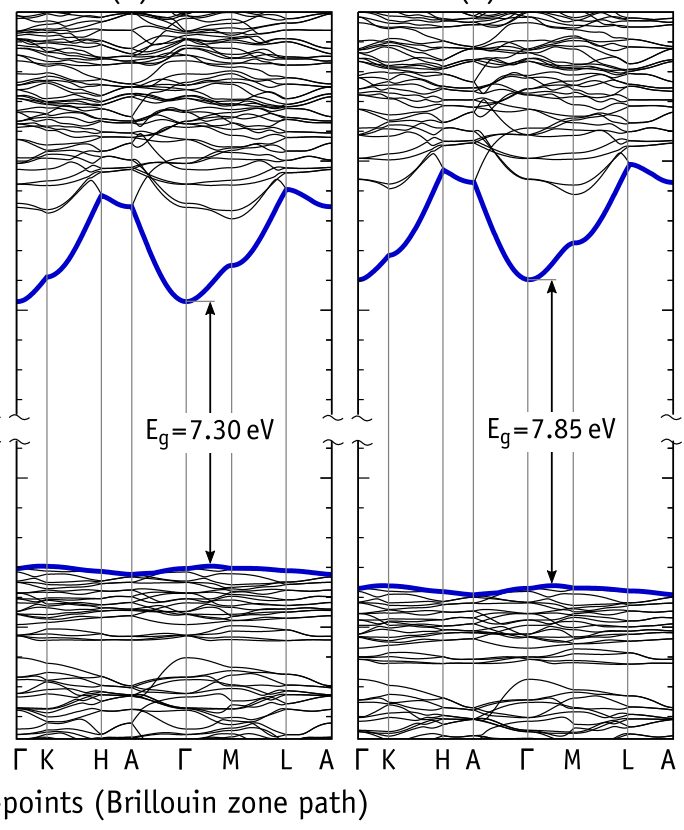

(d) PBEO (e) $G_{0} W_{0}$

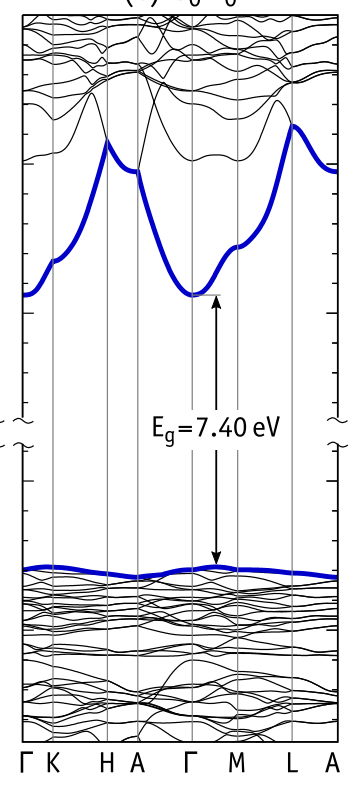

Figure 3. Electronic band structure of bulk HAp along a path with kinks at high-symmetry k-points. Calculations were carried out using DFT within (a) the PBE generalized gradient approximation, hybrid (b) HSE06, (c) B3LYP and (d) PBE0 functionals, as well as (e) using the many-body perturbation $G_{0} W_{0}$ method employing the PBE wave functions. Valence band maximum and conduction band minimum states are represented as thick lines. Indirect band gap widths, $E_{\mathrm{g}}$, are also shown for each case.

hybrid functionals has implications not only to the accuracy of calculated defect-related or inter-band transitions (e.g. observed in luminescence or UV-VIS absorption), but also to transitions involving core or vacuum states (e.g. observed in electron photoemission or core electron energy loss spectroscopy).

The gap of the B3LYP band structure depicted in Figure $3(\mathrm{c})$ is $0.6 \mathrm{eV}$ narrower than that reported by Corno et $a l .{ }^{34}$ using the same functional. Again, the difference is likely to be due to the unsuitability of the atomic-like basis employed in Ref. 34, which resulted in the underscreening of the band structure. The band gap width as well as the band gap edge energies obtained at the B3LYP-level are closer to the $G_{0} W_{0}$ results than any other functional.

The trend of the calculated band gaps agrees with that obtained by Garza and Scuseria ${ }^{77}$ for an eclectic mix of semiconductors and insulators. Accordingly, HSE06 and B3LYP showed a closer correlation with the experiments (the former giving slightly smaller gaps overall), whereas inclusion of larger fractions of exact exchange like in PBE0, led to an overestimation of $E_{\mathrm{g}}$. This ordering also suggests that the gap width obtained within $G_{0} W_{0}\left(E_{\mathrm{g}}=7.4 \mathrm{eV}\right)$ should be close to the real figure. It is also interesting to note that the frequency-dependent screening of the electronic interactions within $G_{0} W_{0}$ has a larger impact on empty states, where several neardegeneracies obtained at DFT level, become well separated when using the many-body perturbation method (see Figure 3(e)).

Figure 4(a) shows the total density of states (DOS) of a HAp unit cell obtained within PBE. The energy scale is directly comparable to the band structure of Figure 3(a). The shadow plots on subsequent Figs. 4(b)-(e), depict the local density of states (LDOS) projected on several atomic species. Along with the LDOS of Figs. 4(b)-(d), we plot a thick line representing the dominant angularmomentum component for the corresponding species. In Fig. 4(e) we distinguish states projected on $\mathrm{O}_{\mathrm{IV}}$ and $\mathrm{H}$ atoms that form $\mathrm{OH}$ molecules. The LDOS calculations considered a Wigner-Seitz projection radius of $1.75 \AA$, $1.23 \AA, 0.82 \AA$ and $0.37 \AA$ for $\mathrm{Ca}, \mathrm{P}, \mathrm{O}$ and $\mathrm{H}$ respectively. For the sake of clearness, the data was convoluted using $0.1 \mathrm{eV}$ wide Lorentzian functions.

At first glance, Figs. 4(b) and 4(c) suggest that the upper end of the valence band is mostly made of $\mathrm{O}(2 \mathrm{p})$ states, while the conduction band bottom is mostly made of $\mathrm{Ca}(3 \mathrm{~d})$ states. From Figs. 4(c) and 4(d) we find phosphorous $3 \mathrm{~s}-3 \mathrm{p}$ states mixing with oxygen $2 \mathrm{~s}-2 \mathrm{p}$ states between -5 and $-2 \mathrm{eV}$, and they are far below from the band gap region. These results are in line with previous reports. $^{23,24}$

Regarding the origin of the bottom of the conduction band, in particular the highly dispersive bands shown in Fig. 3, the situation is more controversial. In Ref. 24, a close analysis of the LDOS at the conduction bottom indicated that the lowest energy bands originated from $\mathrm{Ca}(4 \mathrm{~s})$ states. We argue that this view finds several diffi- 


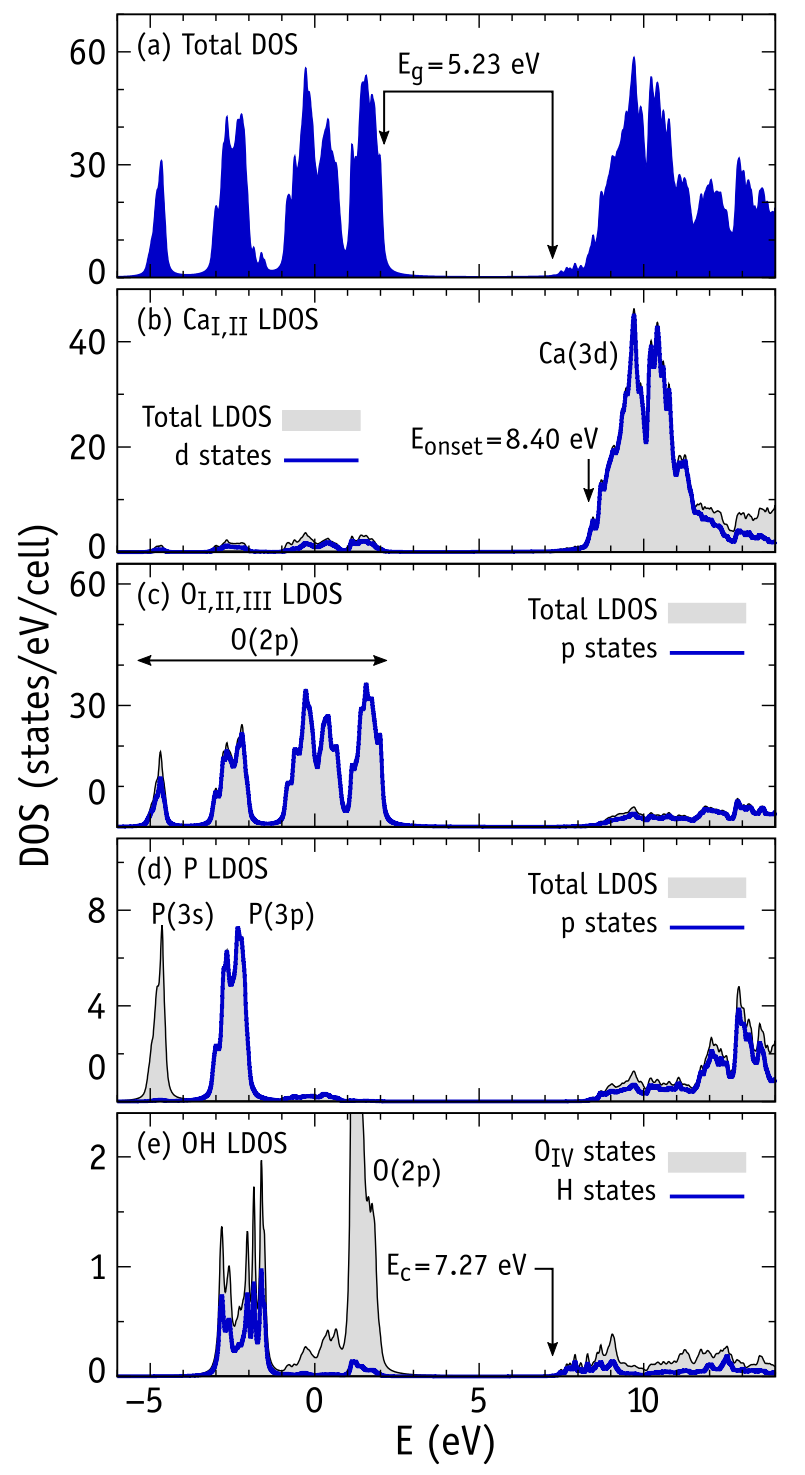

Figure 4. Total density of states of bulk HAp (a) and local densities of states projected on calcium (b), oxygen in $\mathrm{PO}_{4}$ units (c), phosphorous (d), as well as oxygen and hydrogen in $\mathrm{OH}$ units (e). In (b)-(d) the contribution to the LDOS with the dominant angular momentum is represented as a thick line. The results were obtained using the PBE exchangecorrelation functional.

culties. Firstly because we find the onset of the Ca-LDOS $\left(E_{\text {onset }}=8.40 \mathrm{eV}\right)$ located $\sim 1 \mathrm{eV}$ above the conduction band minimum energy. Within that energy range, all that can be related to $\mathrm{Ca}$ are the flat $\mathrm{Ca}(3 \mathrm{~d})$ bands above $8.4 \mathrm{eV}$. Secondly, Figure 4(e) shows that states just above $E_{\mathrm{c}}$ have a considerable localization on $\mathrm{OH}$ molecules. Interestingly, we found that the highly dispersive bands that form the bottom of the conduction band of HAp, are anti-bonding states from an infinite $\cdots \mathrm{OH}-\mathrm{OH}-\cdots$ hydrogen bridge sequence, much like a $1 \mathrm{D}$-ice phase. This statement finds support in the band structure of hexagonal ice (see for example Figures 4 and 6 of Refs. 78 and 79,

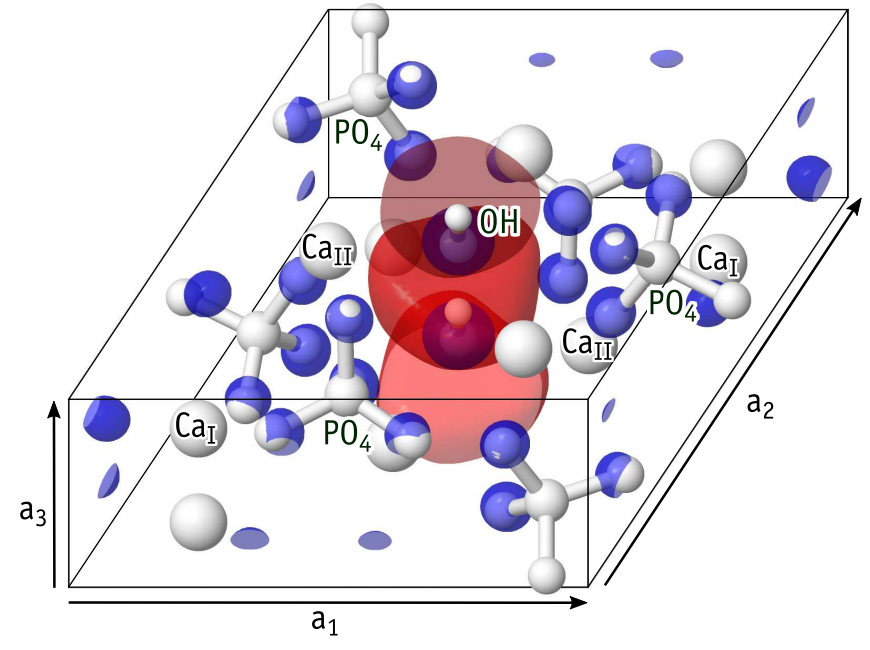

Figure 5. Lowest unoccupied Kohn-Sham state (bottom of the conduction band) of a HAp at $\mathbf{k}=\Gamma$. Blue and red isosurfaces represent $\psi(\mathbf{r})=+0.02$ and $\psi(\mathbf{r})=-0.02$ phases of the wave function. All atoms are shown in white.

respectively). From comparison with our Figure 3(a), it becomes immediately evident that the dispersion shape of the lowest conduction band of HAp, is analogous to that of hexagonal ice.

Finally, our view is demonstrated by Figure 5, which depicts the lowest unoccupied Kohn-Sham state of a HAp at $\mathbf{k}=\Gamma$, using $\psi(\mathbf{r})= \pm 0.02$ isosurfaces. Positive and negative phases are shown in blue and red, respectively. It is unquestionable that the conduction band bottom of HAp is made of anti-bonding $\sigma^{*}$ states along the $\mathrm{OH}$ linear chain, with a minor localization on oxygen atoms of $\mathrm{PO}_{4}$ units, and even less on Ca. The diffuse nature of this state makes it difficult to be projected into atomiclike orbitals, and that could be the reason behind the misassignment in Ref. 24. This finding may have important consequences for doping strategies. For instance, among the possible atomic sites for doping, the replacement of calcium by a foreign species is likely to have the least disruptive effect on the conductive properties of the $\mathrm{OH}$ chain.

\section{Optical properties}

Figure 6 shows the real and imaginary parts of the ion-clamped dielectric function calculated using density functional perturbation theory. For the sake of clearness, we only report the parallel component of $\epsilon_{\infty}$. The basal component was essentially identical to the axial component. The $G_{0} W_{0}$ response curves were obtained employing the quasi-particle corrected band structure.

The shape of the dielectric function obtained using the PBE exchange-correlation functional (tick dashed line) is in general close to that reported by Rulis et al. ${ }^{22}$ obtained within the LDA. The imaginary part, $\epsilon_{\infty}^{(2)}$, is proportional 


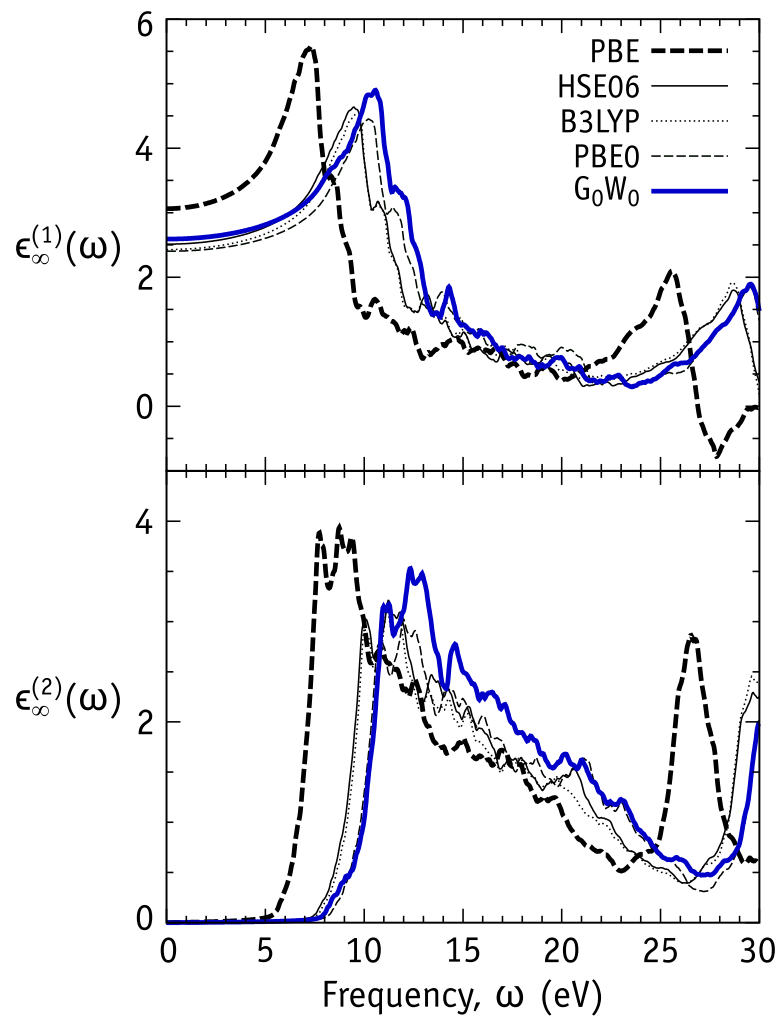

Figure 6. (a) Real part $\epsilon_{\infty}^{(1)}$ and (b) imaginary part $\epsilon_{\infty}^{(2)}$ of the ion-clamped frequency-dependent dielectric function obtained using PBE, HSE06, B3LYP, PBE0 functionals, as well as using the $G_{0} W_{0}$ quasi-particle energies.

to the absorption coefficient due to inter-band transitions. This allows us to obtain some details of $\epsilon_{\infty}(\omega)$ from inspection of the band structure and DOS/LDOS plots of Figures 3 and 4. Accordingly, the onset of $\epsilon_{\infty}^{(2)}$ takes place at about $5.2 \mathrm{eV}$, consistent with the PBE band gap, and therefore to the fundamental transition from the valence band top and the OH-related conduction band bottom. The electronic contribution to the static dielectric constant is $\epsilon_{\infty}^{(1)}(\omega \rightarrow 0) \approx 3$, consistent with $\epsilon_{\infty}=2.93$ as obtained from the calculated electronic polarization upon application of a constant electric field (see Table I).

The first major pole in the dielectric function is located at about $8 \mathrm{eV}$. It results from transitions from the topmost $\mathrm{O}(2 \mathrm{p})$ states shown between $1-2 \mathrm{eV}$ in the DOS plot, to the $\mathrm{Ca}(3 \mathrm{~d})$-related bands. The structure displayed in $\epsilon_{\infty}^{(2)}$ below $15 \mathrm{eV}$ arises from transitions involving the several $\mathrm{O}(2 \mathrm{p})$ bands in the valence to the broad $\mathrm{Ca}(3 \mathrm{~d}) \mathrm{DOS}$ (see also Fig. 4). The second prominent pole at about $27 \mathrm{eV}$ arises from internal $\mathrm{Ca}(3 \mathrm{p}) \rightarrow \mathrm{Ca}(3 \mathrm{~d})$ transitions.

Regarding the results obtained with the hybrid functionals, three main differences are seen when compared to PBE. First, the static dielectric constant using the hybrid functionals decreases by about 0.5 . Within hybrid-DFT we obtain $\epsilon_{\infty}^{(1)}(\omega \rightarrow 0) \approx 2.5$, close to $\epsilon_{\infty}=2.7$ from the
PEAD/hybrid-DFT method in Section III A, but below $\epsilon_{\infty}^{(1)}(\omega \rightarrow 0) \approx 3$ using the PBE approximation. Secondly, all hybrid functionals lead to a blue shift of the dielectric function by 2-3 eV, consistent with an increase of $E_{\mathrm{g}}$ from $5.23 \mathrm{eV}$ to about $7.5 \mathrm{eV}$. The magnitude of this effect is about the same for all hybrid functionals, although slightly more pronounced for PBE0. In fact, PBE0 leads to results closer to those that use the quasi-particle band structure from $G_{0} W_{0}$ calculations. Finally, both real and imaginary parts of the dielectric function show a lower amplitude when using hybrid functionals and the quasiparticle band structure. This follows from the relatively less "crowded" conduction bands when compared to those obtained with PBE (see Figure 3).

\section{Defect formation energies: The $\mathrm{OH}$-vacancy}

We now turn to the assessment of the exchangecorrelation treatment in the calculation of defect formation energies and their electronic levels. This was done by looking at a simple point defect, namely the $\mathrm{OH}$-vacancy (hereafter referred to as $\mathrm{V}_{\mathrm{OH}}$ ). We used 352-atom HAp supercells by replicating $2 \times 2 \times 2=8$ hexagonal unit cells, and removing a single hydroxyl unit to create a $\mathrm{V}_{\mathrm{OH}}$ defect. By doubling the size of the cell along all principal directions, the reciprocal lattice vectors are contracted by a factor of two, so that a $1 \times 1 \times 2$ grid of $\mathbf{k}$-points (to construct the density) would even provide us better sampling quality than the $2 \times 2 \times 3$ grid used for the primitive cell. Convergence tests showed that the total energy of the 352-atom bulk supercell with $\Gamma$-point sampling only, differs by less than $0.1 \mathrm{eV}(0.3 \mathrm{meV} /$ atom $)$ from a $1 \times 1 \times 2$ sampled calculation. Therefore we employed a $\Gamma$-point sampling for defective supercells.

Due to the dramatic impact of the exact exchange treatment in the band structure, the use of hybrid functionals should have important repercussions on the calculated formation energies, particularly on the accuracy of the calculated electronic transition levels. From a thermodynamic perspective, a $E(q / q+1)$ transition level is the location of the Fermi energy within the gap, for which equal populations of defects in charge states $q$ and $q+1$ are found in equilibrium. This takes place when formation energies of both charge states (calculated with help of Eq. 11) are equal for a particular value of $E_{\mathrm{F}}$.

We found that the band structure of HAp with a $\mathrm{V}_{\mathrm{OH}}$ defect differs from that of bulk, only by the presence of an additional semi-occupied defect-related band close to mid-gap. This suggests that $\mathrm{V}_{\mathrm{OH}}$ can donate an electron (leaving the defect band empty) or accept and electron (filling up the defect band). Figure 7 depicts the formation energy of $\mathrm{V}_{\mathrm{OH}}^{q}$ as a function of the Fermi energy, where $q$ is the defect charge state. Positive-, zero- and negative-slope lines represent formation energies of positively charged, neutral and negatively charged vacancies $\left(\mathrm{V}_{\mathrm{OH}}^{+}, \mathrm{V}_{\mathrm{OH}}^{0}\right.$ and $\left.\mathrm{V}_{\mathrm{OH}}^{-}\right)$, respectively. The thick solid lines are the results obtained within PBE-level, while the thin 


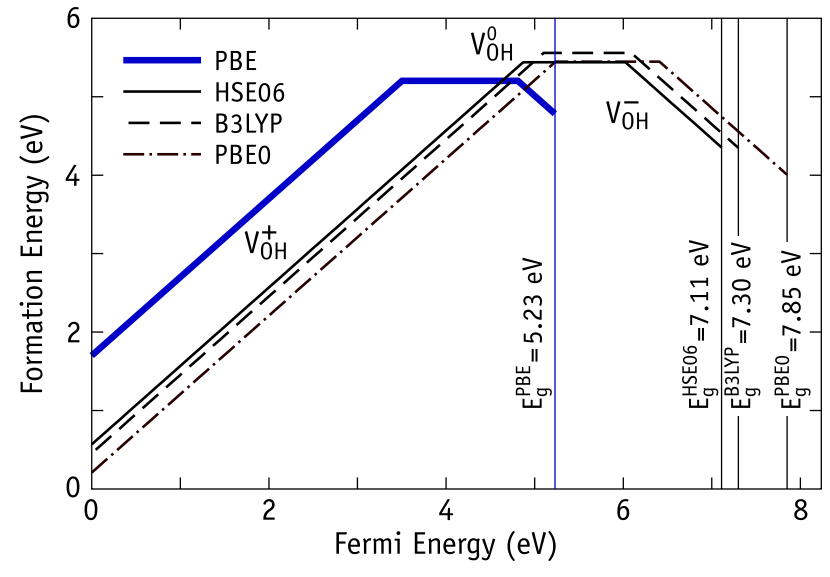

Figure 7. Calculated formation energy of $\mathrm{V}_{\mathrm{OH}}$ in $\mathrm{HAp}$ as a function of the Fermi energy, using different approximations to the exchange-correlation interactions. Positive, neutral and negative charge states are represented by lines with positive, zero and negative slope, respectively. For each functional, the Fermi energy can vary between the valence band top (at $E_{\mathrm{F}}=0 \mathrm{eV}$ ) and the respective conduction band minimum $\left(E_{\mathrm{F}}=E_{\mathrm{g}}\right)$. Chemical potentials of $\mathrm{O}$ and $\mathrm{H}$ were obtained from $\mathrm{O}_{2}$ and $\mathrm{H}_{2}$ molecules.

lines represent formation energies obtained using the hybrid functionals (HSE06, B3LYP and PBE0). For each case, the Fermi energy can vary between $E_{\mathrm{F}}=0$ (for $p$-type material) and $E=E_{\mathrm{g}}$ (for $n$-type material), with $E_{\mathrm{g}}$ being the respective band gap width.

The values of $E_{\mathrm{F}}$ at the "kinks" joining the line segments define the $E(q / q+1)$ transitions. Hence, within $\mathrm{PBE}$ we obtain donor and acceptor transitions at $E(0 /+)=E_{\mathrm{v}}+3.50 \mathrm{eV}$ and $E(-/ 0)=E_{\mathrm{v}}+4.81 \mathrm{eV}$, respectively. On the other hand, using the hybrid functionals we have donor and acceptor transitions at $E(0 /+)=$ $E_{\mathrm{v}}+(5.1 \pm 0.2) \mathrm{eV}$ and $E(-/ 0)=E_{\mathrm{v}}+(6.2 \pm 0.3) \mathrm{eV}$, respectively. The upper and lower limits are obtained using HSE06 and PBE0, respectively. The average values are close to the B3LYP results and differ from the PBE results by about $1.5 \mathrm{eV}$. This is considerably less than the corresponding change in $E_{\mathrm{g}}$, suggesting that the error of (semi)local-functional calculations of electronic transitions, depends much on the character of the defect state that is being populated or emptied. It is also interesting to note that the formation energy of neutral $\mathrm{V}_{\mathrm{OH}}$ using the hybrid functionals is only $0.2 \mathrm{eV}$ above the PBE result. However, there is no reason to conclude that we have found a generality for neutral defects. We assume that this small difference was simply fortuitous.

\section{E. Discussion on corrections to charged defects}

As noted in the last paragraph of Section II D, total energies of charged defects were subject a charge correction $E_{\text {corr }}$ to remove the effect of artificial Coulomb interactions between periodic charged replicas. A question that arises during the calculation of $E_{\text {corr }}$ is - should we use the electronic screening constant $\epsilon_{\infty}$ or the total static dielectric constant $\epsilon_{\mathrm{S}}$ ? As suggested by Komsa et al. ${ }^{80}$, for the calculations of unrelaxed defects (where ligand atoms occupy perfect crystallographic sites), it is appropriate to use the high-frequency (ion-clamped) dielectric constant for describing the screening. However, when atoms are allowed to relax and polarize the supercell, the static low-frequency dielectric constant $\left(\epsilon_{\mathrm{S}}\right)$ should be employed instead.

The above can be verified with help of Figure 8, were we plot the formation energy of both fully relaxed and unrelaxed $\mathrm{V}_{\mathrm{OH}}^{+}$and $\mathrm{V}_{\mathrm{OH}}^{-}$defects, considering $E_{\mathrm{F}}=0$. All data were obtained within PBE level. Tests on HAp unitcells and $2 \times 2 \times 2$ supercells using B3LYP indicated that the dependence on the particular exchangecorrelation functional was negligible. Each plot contains either (i) uncorrected formation energies (shown as crosses), (ii) formation energies corrected by the simple point-charge scheme proposed by Makov and Payne ${ }^{68}$ (shown as squares), and (iii) formation energies corrected by using the FNV method. ${ }^{69,70}$ The effect of the screening constant on the results can be examined from the difference obtained using either the ion-clamped $\epsilon=\epsilon_{\infty} \approx 3$, or the total static dielectric constant $\epsilon=\epsilon_{\mathrm{s}} \approx 11$ (open and solid symbols, respectively). The results are shown as a function of the inverse of the basal dimension of the supercell (lower horizontal axis) and as function of the number of unit cells replicated along each lattice direction (upper horizontal axis). The largest supercell employed had 1188 atoms in bulk, being obtained by replication $3 \times 3 \times 3$ of unit cells along all crystallographic directions. The uncorrected data is used to expand the formation energy in a power series $E(a)=E_{0}+E_{1} a^{-1}+E_{3} a^{-3}$ as proposed in Ref. 81. The second and third terms account for the point-charge-like and dipole-like dependence of the Coulomb correction, respectively. The fit allows us to obtain the formation energy extrapolated to an infinite cell where the charge correction vanishes (horizontal thin line).

It is clear that the correction to be made strongly depends on the defect and its charge state. For the $2 \times 2 \times 2$ supercells used to obtain the data of Figure 7 we have $E_{\text {corr }}$ values of about $0.2 \mathrm{eV}$ and $0.3 \mathrm{eV}$ for positively and negatively charged defects. Although they are a small fraction of $E_{\mathrm{g}}$, they are expected to be 4 times larger for double positive or double negative charge states. This is because the leading term of the correction scales as $E_{\text {corr }} \sim q^{2}$. Another feature, which is well known in the literature (see for instance Refs. 69 and 70), is the fact that the simple point-charge scheme tends to over-estimate the correction. In that respect, the FNV method performs better.

Comparing the plots on the left (unrelaxed) and on the right (relaxed) of Figure 8, we realize that for the unrelaxed cells, the methods that employ the ion-clamped dielectric constant $\left(\epsilon_{\infty}\right)$ are more accurate than using the static dielectric constant $\left(\epsilon_{\mathrm{s}}\right)$. On the other hand, for the 


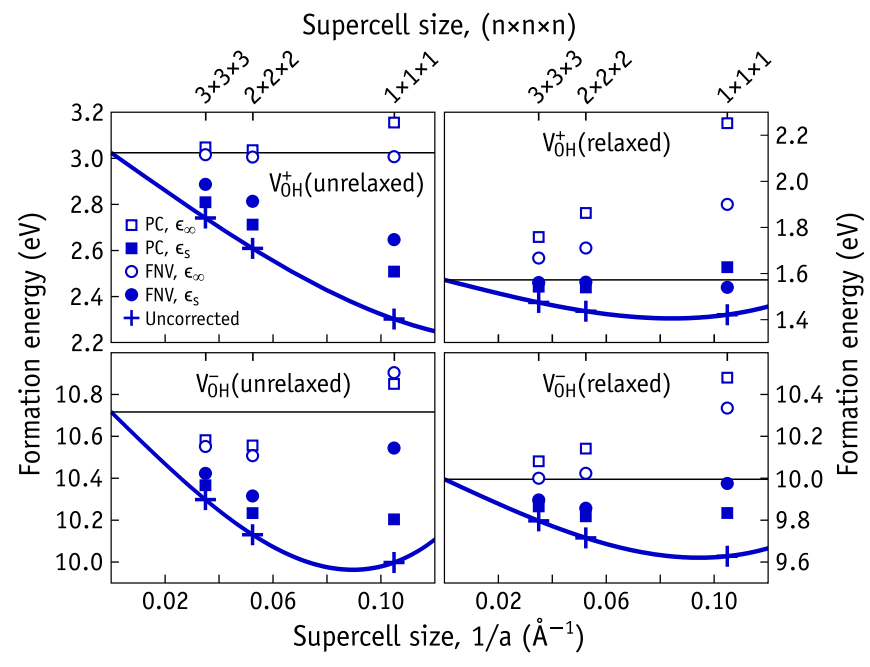

Figure 8. Formation energy of $\mathrm{V}_{\mathrm{OH}}^{+}$and $\mathrm{V}_{\mathrm{OH}}^{-}$in relaxed and unrelaxed supercells, assuming $E_{\mathrm{F}}=0$. Different charge correction schemes are compared on each plot, namely without any charge correction (crosses), with a simple point-charge correction (squares), and with the FNV correction scheme. Results obtained with the ion-clamped and total static dielectric constants are shown as open and solid symbols. Data in the figure were calculated within PBE level. See text for further details.

relaxed cells we obtain an opposite behavior. This shows that the calculation of defect formation energies which involve the relaxation of the atomic positions, should employ the static dielectric constant for the evaluation of the charge correction.

\section{CONCLUSIONS}

We report on the impact of the exchange-correlation treatment to the calculation of several properties of pristine and defective hydroxyapatite. The work was carried out using density functional theory, employing the PBE scheme for the GGA, as well as three popular hybridfunctionals which mix the semi-local exchange with exact Fock exchange. The hybrid-functionals considered are the HSE06, B3LYP and PBE0, and their performance is compared to state-of-the-art many-body perturbation calculations, where the self-energy is accounted for within the $G W$ approximation.

We start by discussing how well the different exchangecorrelation treatments describe the fundamental structural and mechanical properties of HAp. We found that all hybrid-functionals outperform the PBE method, but still, they overestimate the experimental lattice parameters by about $1 \%$, while bulk modulus are underestimated by $3-6 \%$.

The static dielectric response of HAp was investigated by the polarization expansion after discretization method. The electronic contribution to the static dielectric constant was found approximately isotropic. A value of $\epsilon_{\infty}=2.7$ was obtained, irrespectively of the choice of the hybrid-functional. This overestimates the PBE value by 0.2 only, suggesting that the semi-local exchange treatment already provides a reasonable description of the ground state density. We also show that most screening and anisotropy effects are related to the polarization of the lattice by phonons. The components of the static dielectric constant along the basal plane and along the main crystallographic axis, are anticipated as $\epsilon_{\mathrm{s}, \perp}=12.8$ and $\epsilon_{\mathrm{s}, \|}=8.2$. According to the effective medium approximation, these figures correspond to an isotropic dielectric constant $\epsilon_{\mathrm{EMA}}=11$, which is within the observed range of 6-14 from powder samples.

The electronic band structure within PBE is in line with previous equivalent calculations. While the valence band top is made of highly localized $\mathrm{O}(2 \mathrm{p})$ states, the conduction band bottom of HAp is delocalized and shows strong dispersion. These overlap the periodic ... OH-OH- . . hydrogen bridge chain and carry an antibonding $\sigma^{*}$ character, suggesting the formation of a 1Dice phase. This property could be used to explore electrical transport in n-type doped HAp, or for applications involving photo-current. The use of hybrid-DFT was found to have a huge impact on both valence band and conduction band energies. It led to an increase of the band gap width by more than $30 \%$ when compared to PBE results $(5.23 \mathrm{eV})$. The $G W$ quasi-particle band gap was $7.4 \mathrm{eV}$ wide, and the closest hybrid-DFT figure was obtained within B3LYP $(7.3 \mathrm{eV})$. We conclude that (semi-)local calculations of many properties such as defect-related or inter-band transitions (defect levels, UV-VIS spectra), as well as transitions involving core or vacuum states (energy loss or photo-emission spectroscopy) can be largely improved by mixing a portion of exact exchange into the density functional.

Regarding the optical properties, the dielectric function obtained from hybrid-DFT and $G W$ band structure was blue-shifted with respect to the PBE result by $2-3 \mathrm{eV}$. This is consistent with the increase of $E_{\mathrm{g}}$ from $5.23 \mathrm{eV}$ to about $7.5 \mathrm{eV}$ using PBE and hybrid-DFT/ $G W$ methods, respectively. Both real and imaginary parts of the dielectric function show a lower amplitude when using hybrid functionals and the quasi-particle band structure. The PBE0 leads to results closer to the $G W$ calculations.

The choice of the exchange-correlation functional and its impact on the calculation of defect levels was also investigated. We used the $\mathrm{OH}$-vacancy as a testing-model to look at donor and acceptor levels. The levels obtained within hybrid-DFT were markedly different from the PBE results and the difference was not the same for all levels. We finally conclude that any accurate estimation of defect levels using HAp supercells, will require the introduction of a charge correction. This removes spurious interactions between periodic charged replicas from the total energy. The methods available invariably make use of a dielectric constant. It is emphasized that atomic relaxations around the defect polarize the material beyond the ion-clamped level. Hence, the static dielectric 
constant to be used must include both electronic and ionic polarization effects.

\section{ACKNOWLEDGEMENTS}

This work was supported by the Fundação para a Ciência e a Tecnologia (FCT) through project UID $/ \mathrm{CTM} / 50025 / 2013$ and by the Russian Foundation for Basic Research (RFBR) through project No. 15-0104924. SÖ acknowledges the Swedish National Infrastructure for Computing (SNIC) at PDC for providing computational resources.

\section{REFERENCES}

${ }^{1} \mathrm{~V}$. Bystrov, Mathematical Biology and Bioinformatics 12, 14 (2017).

${ }^{2}$ T. Duminis, S. Shahid, and R. G. Hill, Front. Mater. 3, 59 (2017).

${ }^{3}$ B. D. Ratner, A. S. Hoffman, F. J. Schoen, and J. E. Lemons, Biomaterials Science (Elsevier Science, 2004).

${ }^{4}$ M. Epple, K. Ganesan, R. Heumann, J. Klesing, A. Kovtun, S. Neumann, and V. Sokolova, J. Mater. Chem. 20, 18 (2010).

${ }^{5}$ V. S. Bystrov, E. Paramonova, Y. Dekhtyar, A. Katashev,

A. Karlov, N. Polyaka, A. Bystrova, A. Patmalnieks, and

A. Kholkin, J. Phys.: Condens. Matter 23, 065302 (2011).

${ }^{6}$ H. Nishikawa and K. Omamiuda, J. Mol. Catal. A: Chem. 179, 193 (2002).

${ }^{7}$ V. S. Bystrov, J. Coutinho, A. V. Bystrova, Y. D. Dekhtyar, R. C. Pullar, A. Poronin, E. Palcevskis, A. Dindune, B. Alkan, C. Durucan, and E. V. Paramonova, J. Phys. D: Appl. Phys. 48, 195302 (2015).

${ }^{8}$ S. Hu, F. Jia, C. Marinescu, F. Cimpoesu, Y. Qi, Y. Tao, A. Stroppa, and W. Ren, RSC Adv. 7, 21375 (2017).

${ }^{9}$ C. Piccirillo and P. L. Castro, J. Environ. Manage. 193, 79 (2017).

${ }^{10}$ H. Nishikawa, J. Mol. Catal. A: Chem. 207, 149 (2004).

${ }^{11}$ H. Nishikawa, S. Kato, and T. Ando, J. Mol. Catal. A: Chem. 236, 145 (2005).

${ }^{12}$ K. Ozeki, J. M. Janurudin, H. Aoki, and Y. Fukui, Appl. Surf. Sci. 253, 3397 (2007).

${ }^{13}$ M. P. Reddy, A. Venugopal, and M. Subrahmanyam, Appl. Catal., B 69, 164 (2007).

${ }^{14}$ J. H. Shariffuddin, M. I. Jones, and D. A. Patterson, Chem. Eng. Res. Des. 91, 1693 (2013).

${ }^{15}$ E. M. Brazón, C. Piccirillo, I. Moreira, and P. Castro, J. Environ. Manage. 182, 486 (2016).

${ }^{16}$ T. Anirudhan, J. Deepa, and A. S. Nair, J. Ind. Eng. Chem. 47, 415 (2017).

${ }^{17}$ H. Nishikawa, Mater. Lett. 58, 14 (2004).

${ }^{18}$ V. Bystrov, C. Piccirillo, D. Tobaldi, P. Castro, J. Coutinho, S. Kopyl, and R. Pullar, Appl. Catal., B 196, 100 (2016).

${ }^{19}$ G. Rosenman, D. Aronov, L. Oster, J. Haddad, G. Mezinskis, I. Pavlovska, M. Chaikina, and A. Karlov, J. Lumin. 122-123, 936 (2007), luminescence and Optical Spectroscopy of Condensed MatterProceedings of the 2005 International Conference on Luminescence and Optical Spectroscopy of Condensed Matter2005 International Conference on Luminescence and Optical Spectroscopy of Condensed Matter.

${ }^{20}$ M. Tsukada, M. Wakamura, N. Yoshida, and T. Watanabe, J. Mol. Catal. A: Chem. 338, 18 (2011).

${ }^{21}$ L. Calderin, M. Stott, and A. Rubio, Phys. Rev. B. , 134 (2003).

${ }^{22}$ P. Rulis, L. Ouyang, and W. Y. Ching, Phys. Rev. B 70, 155104 (2004).
${ }^{23}$ K. Matsunaga and A. Kuwabara, Phys. Rev. B 75, 014102 (2007).

${ }^{24}$ A. Slepko and A. A. Demkov, Phys. Rev. B 84, 134108 (2011).

${ }^{25} \mathrm{R}$. Martin, L. Reining, and D. Ceperley, Interacting Electrons (Cambridge University Press, 2016).

${ }^{26}$ C. Lee, W. Yang, and R. G. Parr, Phys. Rev. B 37, 785 (1988).

${ }^{27}$ A. D. Becke, J. Chem. Phys. 98, 1372 (1993).

${ }^{28}$ P. J. Stephens, F. J. Devlin, C. F. Chabalowski, and M. J. Frisch, J. Phys. Chem. 98, 11623 (1994).

${ }^{29}$ C. Adamo and V. Barone, J. Chem. Phys. 110, 6158 (1999).

${ }^{30}$ J. Heyd, G. E. Scuseria, and M. Ernzerhof, J. Chem. Phys. 118, 8207 (2003).

${ }^{31}$ A. V. Krukau, O. A. Vydrov, A. F. Izmaylov, and G. E. Scuseria, J. Chem. Phys. 125, 224106 (2006).

${ }^{32}$ G.-X. Qian, R. M. Martin, and D. J. Chadi, Phys. Rev. B 38 , 7649 (1988).

${ }^{33}$ A. Resende, R. Jones, S. Öberg, and P. R. Briddon, Phys. Rev. Lett. 82, 2111 (1999).

${ }^{34}$ M. Corno, C. Busco, B. Civalleri, and P. Ugliengo, Phys. Chem. Chem. Phys. 8, 2464 (2006).

${ }^{35}$ L. Hedin, Phys. Rev. 139, A796 (1965).

${ }^{36}$ M. van Schilfgaarde, T. Kotani, and S. Faleev, Phys. Rev. Lett. 96, 226402 (2006).

${ }^{37}$ M. Shishkin, M. Marsman, and G. Kresse, Phys. Rev. Lett. 99, 246403 (2007).

${ }^{38}$ J. Elliott, Structure and Chemistry of the Apatites and Other Calcium Orthophosphates, Studies in Inorganic Chemistry (Elsevier Science, Amsterdam, 2013) p. 404.

${ }^{39}$ K. M. Stadnicka, B. J. Oleksyn, and K. Z. Sokalski, Acta Crystallographica Section A 43, 156 (1987).

${ }^{40}$ M. Kay, R. Young, and A. Posner, Nature 204, 1050 (1964).

${ }^{41}$ J. Elliott, P. Mackie, and R. Young, Science, 1055 (1973).

${ }^{42}$ J. M. Hughes and J. Rakovan, Rev. Mineral. Geochem. 48, 1 (2002).

${ }^{43}$ T. Ikoma, A. Yamazaki, S. Nakamura, and M. Akao, J. Mater. Sci. Lett. 18, 1225 (1999).

${ }^{44}$ G. Kresse and J. Hafner, Phys. Rev. B 47, 558 (1993).

${ }^{45}$ G. Kresse and J. Hafner, Phys. Rev. B 49, 14251 (1994).

${ }^{46}$ G. Kresse and J. Furthmüller, Computational Materials Science 6, 15 (1996).

${ }^{47}$ G. Kresse and J. Furthmüller, Phys. Rev. B 54, 11169 (1996).

${ }^{48}$ P. E. Blöchl, Phys. Rev. B 50, 17953 (1994).

${ }^{49}$ S. Nakamura, H. Takeda, and K. Yamashita, J. Appl. Phys. , 5386 (2001)

${ }^{50}$ J. P. Perdew, K. Burke, and M. Ernzerhof, Phys. Rev. Lett. 77, 3865 (1996).

${ }^{51}$ R. W. Nunes and X. Gonze, Phys. Rev. B 63, 155107 (2001).

${ }^{52}$ R. D. King-Smith and D. Vanderbilt, Phys. Rev. B 47, 1651 (1993).

${ }^{53}$ I. Souza, J. Iniguez, and D. Vanderbilt, Phys. Rev. Lett. 89, 117602 (2002).

${ }^{54} \mathrm{M}$. Born and K. Huang, Dynamical theory of crystal lattices, International series of monographs on physics (Oxford University Press, Oxford, 1954).

${ }^{55}$ E. Cockayne and B. P. Burton, Phys. Rev. B 62, 3735 (2000).

${ }^{56}$ O. Kaygili, S. Keser, T. Ates, A. A. Al-Ghamdi, and F. Yakuphanoglu, Powder Technol. 245, 1 (2013).

${ }^{57}$ T. P. Hoepfner and E. D. Case, J. Biomed. Mater. Res. 60, 643 (2002).

${ }^{58}$ T. G. Mayerhöfer, Appl. Spectrosc. 56, 1194 (2002).

${ }^{59}$ T. G. Mayerhöfer and J. Popp, Icarus 203, 303 (2009).

${ }^{60}$ S. L. Adler, Phys. Rev. 126, 413 (1962).

${ }^{61}$ N. Wiser, Phys. Rev. 129, 62 (1963).

${ }^{62}$ M. Gajdos, K. Hummer, G. Kresse, J. Furthmuller, and F. Bechstedt, Phys. Rev. B 73, 045112 (2006).

${ }^{63}$ M. Shishkin and G. Kresse, Phys. Rev. B 74, 035101 (2006).

${ }^{64}$ F. Fuchs, J. Furthmüller, F. Bechstedt, M. Shishkin, and G. Kresse, Phys. Rev. B 76, 115109 (2007).

${ }^{65}$ F. J. Cruz, M. E. M. da Piedade, and J. C. Calado, The Journal of Chemical Thermodynamics 37, 1061 (2005). 
${ }^{66}$ J. L. Katz and K. Ukraincik, J. Biomech. 4, 221 (1971).

${ }^{67}$ R. S. Gilmore and J. L. Katz, J. Mater. Sci. 17, 1131 (1982).

${ }^{68}$ G. Makov and M. C. Payne, Phys. Rev. B 51, 4014 (1995).

${ }^{69}$ C. Freysoldt, J. Neugebauer, and C. G. Van de Walle, Phys. Rev. Lett. 102, 016402 (2009).

${ }^{70}$ Y. Kumagai and F. Oba, Phys. Rev. B 89, 195205 (2014).

${ }^{71}$ M. E. Zilm, L. Chen, V. Sharma, A. McDannald, M. Jain, R. Ramprasad, and M. Wei, Phys. Chem. Chem. Phys. 18, 16457 (2016).

72 J. Paier, M. Marsman, and G. Kresse, The Journal of Chemical Physics 127, 024103 (2007).

${ }^{73}$ F. Birch, Phys. Rev. 71, 809 (1947).

${ }^{74}$ Z. Yuan, T. Gao, Y. Zheng, S. Ma, M. Yang, and P. Chen, RSC Adv. 7, 30310 (2017).

${ }^{75}$ R. D. Shannon and G. R. Rossman, Physics and Chemistry of Minerals 19, 157 (1992).
76 A. A. Mostofi, J. R. Yates, G. Pizzi, Y.-S. Lee, I. Souza, D. Vanderbilt, and N. Marzari, Comp. Phys. Comm. 185, 2309 (2014).

${ }^{77}$ A. J. Garza and G. E. Scuseria, The Journal of Physical Chemistry Letters 7, 4165 (2016).

${ }^{78}$ D. Prendergast, J. C. Grossman, and G. Galli, The Journal of Chemical Physics 123, 014501 (2005).

${ }^{79}$ E. A. Engel, B. Monserrat, and R. J. Needs, The Journal of Chemical Physics 143, 244708 (2015).

${ }^{80}$ H.-P. Komsa, T. T. Rantala, and A. Pasquarello, Phys. Rev. B 86, 045112 (2012).

${ }^{81}$ C. W. M. Castleton, A. Höglund, and S. Mirbt, Phys. Rev. B 73, 035215 (2006). 